Mathematical Modelling and Analysis

Volume 18 Number 5, November 2013, 708-716

http://dx.doi.org/10.3846/13926292.2013.871651

(c) Vilnius Gediminas Technical University, 2013
Publisher: Taylor\&Francis and VGTU

http://www.tandfonline.com/TMMA

Print ISSN: $1392-6292$

Online ISSN: 1648-3510

\title{
On Periodic Solutions of Liénard Type Equations
}

\section{Svetlana Atslega ${ }^{a}$ and Felix Sadyrbaev ${ }^{b}$}

${ }^{a}$ Latvia University of Agriculture

2 Liela Street, LV-3001 Jelgava, Latvia

${ }^{b}$ Daugavpils University

Parades str. 1, LV-5400 Daugavpils, Latvia

E-mail: felix@latnet.lv

Received December 9, 2011; revised November 29, 2013; published online December 1, 2013

\begin{abstract}
The Liénard type equation $x^{\prime \prime}+f\left(x, x^{\prime}\right) x^{\prime}+g(x)=0(i)$ is considered. We claim that if the associated conservative equation $x^{\prime \prime}+g(x)=0$ has period annuli then a dissipation $f\left(x, x^{\prime}\right)$ exists such that a limit cycle of equation $(i)$ exists in a selected period annulus. Moreover, it is possible to define $f\left(x, x^{\prime}\right)$ so that limit cycles appear in all period annuli. Examples are given. A particular example presents two limit cycles of non-convex shape in two disjoint period annuli.
\end{abstract}

Keywords: multiple solutions, second-order equation, periodic solutions, limit cycles.

AMS Subject Classification: 34B08; 34B15.

\section{Introduction}

Limit cycles are of great importance from theoretical point of view and are useful in applications. Due to famous problems aimed at the existence and localization of limit cycles of two-dimensional systems there is intensive literature on the subject. One may consult the books [3,6,7], article [5] and literature therein. The important special case is the system

$$
x^{\prime}=y, \quad y^{\prime}=-f(x) y-g(x),
$$

which is known as the generalized Liénard equation

$$
x^{\prime \prime}+f(x) x^{\prime}+g(x)=0 .
$$

Limit cycles can exist around the critical points.

If $f$ is a polynomial of high degree then there may be multiple limit cycles around the unique critical point even for a simple $g(x)=x$.

From physical point of view equation (1.1) describes oscillations in a system where dissipation is given by the term $f(x) x^{\prime}$. The respective conservative 
equation

$$
x^{\prime \prime}+g(x)=0
$$

cannot have limit cycles. It can have continua of periodic solutions. These continua form period annuli.

In this article we consider the Liénard type equations

$$
x^{\prime \prime}+f\left(x, x^{\prime}\right) x^{\prime}+g(x)=0,
$$

where $f$ depends also on $x^{\prime}$. We show that in any period annulus of conservative equation (1.2) a limit cycle may emerge under an appropriate dissipation.

We use period annuli of the associated conservative equation (1.2) as topographic systems [7] for equation (1.3).

\section{Period Annuli}

Consider the second order differential equation (1.2). It is supposed that $g(x)$ has simple zeros $p_{i}$, then the respective primitive function has local extrema. The equivalent two-dimensional differential system

$$
x^{\prime}=y, \quad y^{\prime}=-g(x)
$$

has critical points at $\left(p_{i}, 0\right)$, where $p_{i}$ are simple zeros of $g(x)$.

Proposition 1. Critical points of the system (2.1) are "saddle points" and "centers" which alternate.

Proof. When written in a two-dimensional form and linearized, this equation possesses critical points with purely imaginary and real eigenvalues of opposite signs which corresponds to respectively center points and saddle points. Besides, these points alternate due to the fact that zeros of $g(x)$ are simple. Recall that a critical point zero of (2.1) is a center if it has a punctured neighborhood covered with nontrivial cycles.

The two definitions below are in the form of $[8,9]$.

Definition 1. A central region is the largest connected region covered with cycles surrounding zero.

Definition 2. A period annulus is every connected region covered with nontrivial concentric cycles.

Definition 3. We will call a period annulus associated with a central region a trivial period annulus. Periodic trajectories of a trivial period annulus encircle exactly one critical point of the type center.

Definition 4. A period annulus enclosing several (more than one) critical points will be called a nontrivial period annulus. 
Consider differential equation (1.2) where $g(x)$ is of the form

$$
g(x)=-\left(x-x_{1}\right) \cdots\left(x-x_{2 n-1}\right), \quad 2 n-1 \geq 5, x_{1}<\cdots<x_{2 n-1}
$$

Let $G(x)=\int_{0}^{x} g(s) d s$.

Theorem 1. $[\mathbf{1}, \mathbf{2}]$ Let $m_{1}$ and $m_{2}\left(m_{1}<m_{2}\right)$ be non-neighbouring points of maximum of the function $G(x)$. Suppose that any other local maximum of $G(x)$ in the interval $\left(m_{1}, m_{2}\right)$ is strictly less than $\min \left\{G\left(m_{1}\right) ; G\left(m_{2}\right)\right\}$.

Then there exists at least one nontrivial period annulus.

To illustrate period annuli consider the example. Let the equation be

$$
x^{\prime \prime}-(x+2.4)(x+1.8)(x+1) x(x-0.8)(x-1.7)(x-2.4)=0 \text {. }
$$

The primitive $G(x)$ is depicted in Fig. 1a.

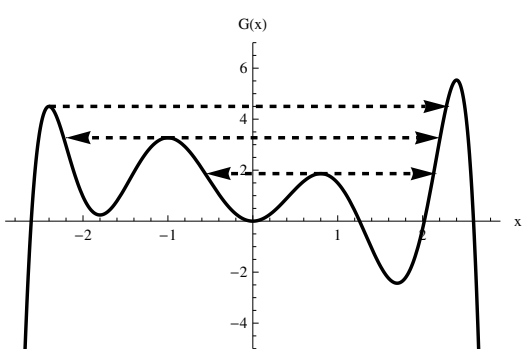

a)

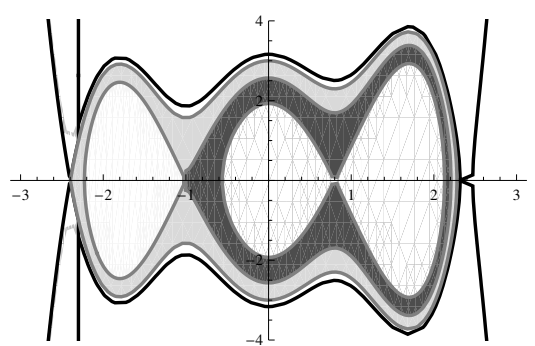

b)

Figure 1. a) The primitive function

$G(x)=-\frac{1}{8} x^{8}-\frac{3}{70} x^{7}+\frac{8}{5} x^{6}+\frac{121}{250} x^{5}-\frac{7677}{1250} x^{4}-\frac{4152}{3125} x^{3}+\frac{22032}{3125} x^{2}$ for $g(x)$ in $\left.(2.3) ; \mathrm{b}\right)$ the
phase plane, there are three trivial and two non-trivial period annuli.

\section{Limit Cycles}

We consider equation (1.3) together with the associated conservative equation (1.2). We assume that there is a period annulus in equation (1.2). To detect a limit cycle in dissipative equation (1.3) we use the classical result [7, Th. 2.8.8].

Theorem 2 [Poincaré-Bendixson Theorem]. Suppose $R$ is the finite region of the plane lying between two simple closed curves $D_{1}$ and $D_{2}$, and $F$ is the velocity vector field for the system

$$
x^{\prime}=f(x, y), \quad y^{\prime}=g(x, y) .
$$

If at each point of $D_{1}$ and $D_{2}$, the field $F$ points toward the interior (or the exterior) of $R$, and $R$ contains no critical points, then the system has a closed trajectory lying inside $R$. 
Definition 5. A limit cycle on plane is a closed trajectory in phase plane having the property that at least one other trajectory spirals into it either as time approaches infinity or as time approaches negative infinity.

The classical example is a limit cycle in Van der Pol equation

$$
x^{\prime \prime}+\mu\left(x^{2}-1\right) x^{\prime}+x=0, \quad \mu>0 .
$$

As $\mu \rightarrow 0$ the limit cycle tends to a circular closed orbit of the limit equation $x^{\prime \prime}+x=0$.

Conservative equation (1.2) cannot have limit cycles, but they can appear in equations of the type (1.3).

Lemma 1. Any period annulus of equation (1.2) (it is defined by the relation $W\left(x, x^{\prime}\right)=2 G(x)+x^{\prime 2}=$ const) is a topographic system for equation (1.3) and the derivative $W^{\prime}$ along trajectories of (1.3) is given by

$$
\frac{d W\left(x(t), x^{\prime}(t)\right)}{d t}=-2 f\left(x(t), x^{\prime}(t)\right) x^{\prime 2}(t)
$$

Proof. The first assertion follows from definition of a topographic system [7, p. 65]. The second one is proved below.

$$
\begin{aligned}
\frac{d W\left(x(t), x^{\prime}(t)\right)}{d t} & =2 x^{\prime}(t) x^{\prime \prime}(t)+2 g(x(t)) x^{\prime}(t) \\
& =2 x^{\prime}(t)\left(-f\left(x(t), x^{\prime}(t)\right) x^{\prime}(t)-g(x(t))\right)+2 g(x(t)) x^{\prime}(t) \\
& =-2 f\left(x(t), x^{\prime}(t)\right) x^{\prime 2}(t) .
\end{aligned}
$$

\subsection{Existence}

Our result is the following theorem.

Theorem 3. Let $P$ be a period annulus in a conservative equation (1.2). Suppose that $f\left(x, x^{\prime}\right)$ in associated equation $(1.3)$ is a $C^{1}\left(R^{2}\right)$ function such that a set of zeros of $f$ in $P$ is a closed Jordan curve $Z$ and $f$ is of opposite signs for $\left(x, x^{\prime}\right)$ inside and outside the region bounded by the curve $Z$. Then equation (1.3) has a limit cycle in $P$.

Proof. Consider a period annulus $P$ of (1.2). Let $C_{1}$ and $C_{2}$ be respectively the inner and outer boundaries of $P$ (if $P$ is the central region then $C_{1}$ is identified with the respective critical point). Denote $D$ the annular region between $C_{1}$ and $C_{2}$. Function $f\left(x, x^{\prime}\right)$ is equal to zero at $\left(x, x^{\prime}\right) \in Z$ and it is of opposite signs for $\left(x, x^{\prime}\right)$ in the interior and the exterior of the region bounded by the curve $Z$.

Consider the dissipative equation (1.3). The annular region $D$ is the Poincaré-Bendixson region with the property that any trajectory of (1.3) which starts at $C_{1}$ or $C_{2}$ enters $D$. The only exceptions are points at intersections of $C_{1}$ or $C_{2}$ with the $x$-axis, where trajectories of (1.3) are vertically tangent to $C_{1}$ and $C_{2}$. 
Consider the segment $\Sigma$ of the $x$-axis lying in the "left" part of $D$. The "left" part is naturally defined in case of a non-trivial period annulus. It is the left part with respect to the central point in case of a central region. Let $x \in \Sigma$. The Poincaré map (or first return map, [6, p. 206]) $P(x)=x-x(T)$ ( $T$ is the "period") has different signs for $x$ near $\Sigma \cap C_{2}$ and $x$ near $\Sigma \cap C_{1}$, due to (3.1). Therefore there exists a limit cycle in $P$.

Remark 1. In fact $f\left(x, x^{\prime}\right)$ may be of opposite signs in distinct components of the set $P \backslash Z$.

Lemma 2. If there are multiple (but finite number of) period annuli in equation (1.2) then a polynomial function $f\left(x, x^{\prime}\right)$ can be defined such that the respective dissipative equation (1.3) has a limit cycle in any period annulus.

Proof. Any period annulus in equation (1.2) can be obtained from the relation

$$
x^{2}+2 G(x)=c, \quad c_{1}<c<c_{2} .
$$

Choose $f_{i}\left(x, x^{\prime}\right)$ as $f_{i}=x^{\prime 2}+2 G(x)-\frac{c_{1}+c_{2}}{2}$. Define $f\left(x, x^{\prime}\right)=\prod_{i} f_{i}\left(x, x^{\prime}\right)$. Obviously $f$ is a polynomial (recall that $g(x)$ and in turn $G(x)$ are polynomials). There is exactly one simple closed curve $Z$ per period annulus. There exists at least one limit cycle in any of period annuli by Theorem 3 .

\subsection{Number of limit cycles}

The number of limit cycles that appear in a Liénard type equations (1.3) is not less than the number of period annuli, both trivial and nontrivial ones, in an associated conservative equation.

Theorem 4. A 2n-1-th degree polynomial $g(x)$ satisfying $(2.2)$ can be chosen so that at least $2 n-3$ limit cycles can be obtained by adding the dissipative member in equation (1.2).

Proof. Let us count the number of period annuli, both trivial (center regions) and nontrivial, in equation (1.2). There are exactly $n-1$ centers since $g(x)$ satisfies the condition (2.2).

Any two non-neighboring points of maxima $m_{1}$ and $m_{2}$ of the function $G(x)=\int_{0}^{x} g(s) d s$, which satisfy the condition of Theorem 1 , form a regular pair (Definition 3.1 in [4]). Accordingly to the result (Theorem 4.1) in [4] $g$ can be chosen in such way that the number of regular pairs of $G$ is exactly $k-2$, where $k$ is the number of maxima of the function $G(x)$. Each regular pair generates a nontrivial period annulus due to Theorem 1 . Therefore the number of nontrivial period annuli in equation (1.2) (where $g$ is the above chosen) is not less than $k-2$ or, in terms of the degree of $g(x)$, not less than $n-2$ (the degree $(2 n-1)+1$ of the primitive function $G(x)$, divided by 2$)$.

Totally there are at least $(n-2)+(n-1)=2 n-3$ period annuli in (1.2), and therefore there are at least $2 n-3$ limit cycles in a Liénard type equations (1.3). 


\subsection{Examples}

Example 1. Consider the conservative equation $x^{\prime \prime}+g(x)=0$, where $g(x)=$ $-x\left(x^{2}-2^{2}\right)$. The primitive $G(x)=2 x^{2}-x^{4} / 4$ has a minimum at $x=0$ (a double zero) and two symmetric maxima at $x= \pm 2$. The respective system has the trivial period annulus around the critical point $(0,0)$.

Consider the Liénard type equation

$$
x^{\prime \prime}=-\left(x^{2}+x^{2} / 4-1\right) x^{\prime}+x\left(x^{2}-4\right) .
$$

As a result, in the trivial period annulus of the equation $x^{\prime \prime}-x\left(x^{2}-2^{2}\right)=0$ emerges a limit cycle of the equation (3.2), see Fig. 2.

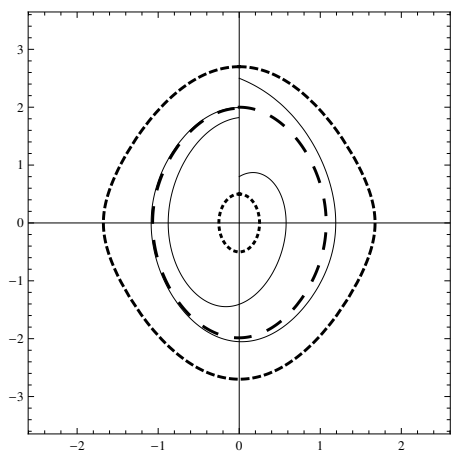

Figure 2. The middle dashed line represents a limit cycle in Example 1, $x(0)=0, x^{\prime}(0)=2.0$. Two solutions are shown tending to the limit cycle, $x(0)=0$, $x^{\prime}(0)=0.8, x(0)=0, x^{\prime}(0)=2.5$. The period annulus of the conservative equation which transforms to the Poincaré-Bendixson region for the Liénard type equation is marked by two dashed closed inner and outer curves.

Example 2. Consider the conservative equation $x^{\prime \prime}+g(x)=0$, where

$$
g(x)=-x\left(x^{2}-1\right)\left(x^{2}-(15 / 10)^{2}\right)\left(x^{2}-(28 / 10)^{2}\right)\left(x^{2}-(346 / 100)^{2}\right) .
$$

The graph of the primitive function

$$
\begin{aligned}
G(x) & =\int_{0}^{x} g(x) d x \\
& =-\frac{13198689}{12500} x^{2}+\frac{87403117}{100000} x^{4}-\frac{13374587}{50000} x^{6}+\frac{28827}{1000} x^{8}-x^{10}
\end{aligned}
$$

is depicted in Fig. 3.

Consider the Liénard type equation

$$
\begin{aligned}
x^{\prime \prime}= & -10\left(\frac{(x-2.8)^{2}}{0.5^{2}}+\frac{x^{2}}{10^{2}}-1\right) x^{\prime} \\
& +x\left(x^{2}-1\right)\left(x^{2}-\left(\frac{15}{10}\right)^{2}\right)\left(x^{2}-\left(\frac{28}{10}\right)^{2}\right)\left(x^{2}-\left(\frac{346}{100}\right)^{2}\right)
\end{aligned}
$$




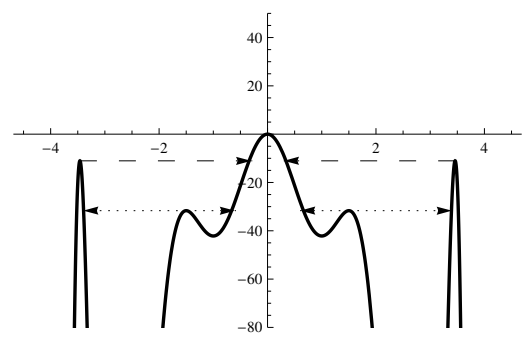

Figure 3. The function $G(x)$ in Example 2. There are two symmetric non-trivial period annuli and two symmetric pairs of trivial period annuli in the respective equation $x^{\prime \prime}+g(x)=0$.

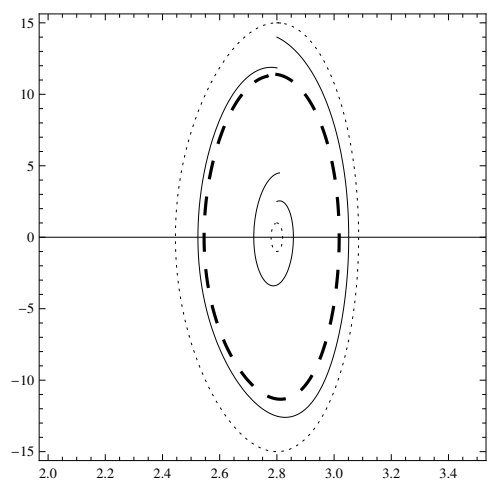

Figure 4. The middle dashed line represents a limit cycle, $x(0)=2.8, x^{\prime}(0)=11.39$. Two solutions are shown tending to the limit cycle, $x(0)=2.8, x^{\prime}(0)=2.5, x(0)=2.8$,

$x^{\prime}(0)=14$. The period annulus of the conservative equation which transforms to the Poincaré-Bendixson region for the Liénard type equation is marked by two dotted closed inner and outer curves.

obtained from the conservative one by adding the middle dissipative term. This equation possesses two limit cycles located in two non-trivial period annuli of the conservative equation.

Figure 4 shows one of the trivial period annulus of the conservative equation (that around the critical point $(2.8,0)$ ) and a limit cycle of the respective dissipative equation inside.

\subsection{Limit cycles of non-convex shape in disjoint nontrivial period annuli}

Consider equation

$$
x^{\prime \prime}+g(x)=0,
$$

where $g(x)$ is the same as in Example 2 and the Liénard type equation

$$
x^{\prime \prime}+\left(2 G(x)+x^{\prime 2}+440\right) x^{\prime}+g(x)=0 .
$$

Two outer solutions (a), two inner solutions (b) and two respective limit cycles (c) are depicted in Fig. 5. 


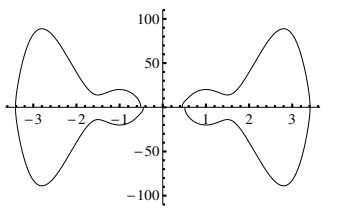

a) $x(0)= \pm 0.45, x^{\prime}(0)=0$;

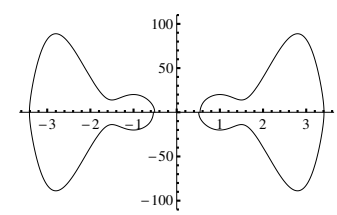

b) $x(0)= \pm 0.54, x^{\prime}(0)=0$;

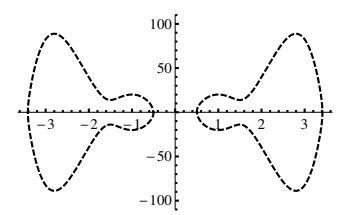

c) $x(0)= \pm 0.5, x^{\prime}(0)=0$.

Figure 5. a) $x(0)= \pm 0.45, x^{\prime}(0)=0$; b) $x(0)= \pm 0.54, x^{\prime}(0)=0$; c) $x(0)= \pm 0.5, x^{\prime}(0)=0$.

\section{Conclusions}

Existence and location of limit cycles in polynomial differential equations are difficult problems. They cannot appear in conservative equations. Conservative equations may have regular continua of periodic solutions which form period annuli in a phase plane. Period annuli may produce limit cycles under perturbation of a conservative equation by dissipative terms. This process can be traced and controlled in case the dissipation is dependent on both phase variables $x$ and $x^{\prime}$. The main step in adding the dissipative member is defining closed curves in period annuli. If there is enough space in a period annulus an ellipse can be inscribed in it thus raising the degree of polynomials in equation by two. If the shape of a period annulus is not simple, then the degree of an inscribed closed curve is higher. Since $g(x)$ is supposed to be polynomial the integral of a conservative equation $x^{\prime \prime}+g(x)=0$ is

$$
2 G(x)+x^{\prime 2}=\text { const, }
$$

which is a polynomial in the left side. Trivially the same degree closed curve can be inscribed in a respective period annulus.

The relation (4.1) may define several period annuli. Consequently a unique relation of the type (4.1) may define several closed curves in multiple period annuli. So the problem arises: to inscribe closed curves in all period annuli by adding to a conservative equation a unique polynomial member $f\left(x, x^{\prime}\right)$ of possibly lower degree.

\section{Acknowledgments}

The authors would like to thank the referees for careful reading of the manuscript and constructive remarks.

\section{References}

[1] S. Atslega and F. Sadyrbaev. Period annuli in the Liénard type equation. Int. J. Pure Appl. Math., 44(1):117-123, 2008.

[2] S. Atslega and F. Sadyrbaev. Multiple solutions of the second order nonlinear Neumann BVP. In Supplement Dedicated to the 6th Intern. Conf. on Differential Equations and Dynamical Systems Held in Baltimore, U.S.A., May 22-26, Dyn. Contin. Discrete Impuls. Syst. Ser. A, pp. 100-103. Watam Press, 2009. 
[3] C. Chicone. Ordinary Differential Equations with Applications. Springer, 1999.

[4] Y. Kozmina and F. Sadyrbaev. On a maximal number of period annuli. Abstr. Appl. Anal., 2011:8 pp., 1997. http://dx.doi.org/10.1155/2011/393875. Available from Internet: http://www.hindawi.com/journals/aaa/2011/393875/. Article ID 393875

[5] J. Llibre and G. Rodríguez. Configurations of limit cycles and planar polynomial vector fields. J. Differential Equations, 198(2):374-380, 2004.

http://dx.doi.org/10.1007/s10625-005-0242-y.

[6] L. Perko. Differential Equations and Dynamical Systems. Third edition. Springer, 2001.

[7] R. Reissig, G. Sansone and R. Conti. Qualitative Theorie Nichtlinear Differentialgleichungen. Edizioni Cremonese, Roma, 1963. Russian translation "Nauka", 1974

[8] M. Sabatini. On the period function of $x^{\prime \prime}+f(x) x^{2}+g(x)=0$. J. Differential Equations, 196(1):151-168, 2004. http://dx.doi.org/10.1016/S0022-0396(03)00067-6.

[9] M. Sabatini. Liénard limit cycles enclosing period annuli, or enclosed by period annuli. Rocky Mountain J. Math., 35(1):253-266, 2005.

http://dx.doi.org/10.1216/rmjm/1181069780. 